\section{Bioethik - Normenbegründung zwischen Naturwissenschaft und Menschenbild}

\author{
Bericht von der Abschlusstagung des \\ Graduiertenkollegs „Bioethik“ am Inter- \\ nationalen Zentrum für Ethik in den Wis- \\ senschaften der Universität Tübingen
}

Tübingen, 7.-8. Februar 2013

\section{von Martin Sand, ITAS}

Die Biowissenschaften haben neben anderen Lebewesen den Menschen zum Gegenstand ihrer Forschung. Sie untersuchen und beschreiben den Menschen oder Teile des Menschen in funktionalen und kausalen Begriffsschemata, als Systeme oder Organismen. Nicht selten wird dieses Bild als vollständige Beschreibung des menschlichen Wesens ausgegeben und stößt damit auf Widerspruch. Es kommt uns zumindest so vor, als würden wir nicht wegen äußerer oder innerer Ursachen so oder so handeln, sondern aus Gründen, die wir gegeneinander abwägen. Solche Entscheidungen scheinen von gar nichts verursacht zu sein, außer vielleicht unserem Willen. Kurzum: Manche Biowissenschaftler vertreten ein naturalistisches Menschenbild und dieses wirft wissenschaftstheoretische, philosophische und ethische Fragen von erheblicher Tragweite auf (Janich 2009).

Die Biowissenschaften entwickelten sich im medizinischen und gentechnischen Anwendungsbereich in den letzten Jahren rasant weiter. Unter welchen Bedingungen, in welchen Kontexten wollen wir diese Anwendungen einsetzen? Wie können wir die Folgen dieser Anwendungen abschätzen? Wie verändern Gentechnik, NeuroEnhancement und Synthetische Biologie unsere „Natur" und unser Zusammenleben (Habermas 2002)? Wer trägt für diese Entwicklungen Verantwortung (Lenk 1991)?

Die Erwartungen, was die Zukunft biotechnologischer Anwendungen angeht, reichen von utopischen Heilsversprechen bis zu apokalyptischen Untergangsszenarien (Saage 2006). Betroffene Personen und Akteure suchen in diesem Zusammenhang nach Orientierung (Grunwald 2008). Seit 2004 beschäftigt sich das von der
Deutschen Forschungsgemeinschaft geförderte Graduiertenkolleg „Bioethik“ unter der Leitung von Eve-Marie Engels und Thomas Potthast mit solchen Fragen. Bei der Abschlusstagung in Tübingen wurden die bisherigen Ergebnisse der Projekte aus der zweiten und dritten Förderphase präsentiert. Man näherte sich der Bioethik von unterschiedlichen Seiten und unterstrich damit die begrüßenswerte interdisziplinäre Ausrichtung des Kollegs. Die Vorträge behandelten u. a. das Verhältnis Darwins zur Eugenik, Transparenzforderungen an die biomedizinischen Forschung, die Brauchbarkeit der Ethik Albert Schweitzers für die Bioethik, das deontologische Moralverständnis und die Natur des Menschen, Menschenbilder in den Erziehungswissenschaften, der Bioethik und Genetik und ethische Fragen der Epigenetik. Die Vorträge nahmen Rücksicht auf den gegenwärtigen Forschungsstand und erreichten durchweg ein hohes Niveau. Im Folgenden kann leider nicht auf alle Ergebnisse eingegangen werden, deswegen werden einige Vorträge ausgewählt.

\section{Deontologie und Menschenbild}

Die erste Präsentation von Daniel Henrich beschäftigte sich mit dem deontologischen Moralverständnis und der Natur des Menschen. Henrich stellte die Frage, ob und wie sich das deontologische Moralverständnis mit dem naturalistischen Menschenbild der Biowissenschaften vereinen lasse. Das deontologische Moralverständnis von Kant geht davon aus, dass der Mensch in der Lage sei, sich selbst ein Gesetz zu geben, respektive moralische Prinzipien zu entwickeln. Am Ende der „Grundlegung der Metaphysik der Sitten“ gelangt Kant zu dem berühmten Schluss, dass der kategorische Imperativ das höchste Prinzip sei, dass man sich denken könne (Patzig 1988). Die Achtung vor diesem höchsten Gesetz gebiete gleichsam seine Berücksichtigung. Henrich argumentierte über Habermas in diese Richtung und bezeichnete die Selbstgesetzgebung als maßgebliches Charakteristikum der menschlichen Natur. Die Möglichkeit der Selbstgesetzgebung hänge mit dem Begriff der Freiheit eng zusammen. Positiv könne Freiheit als Eigenschaft eines spezifischen Wissenstypus, dem der Lebenswelt, interpretiert werden. 
Henrich erläuterte anhand der Philosophien von Edmund Husserl und Peter Janich, dass Freiheit eine immanent lebensweltliche Erfahrung sei.

Eve-Marie Engels kritisierte, dass das Problem der Verschmelzung des wissenschaftlichen, kausalen Verständnisses der menschlichen Natur durch die vorgestellte Konzeption des performativen, lebensweltlichen Freiheitsbegriffs gerade nicht gelöst würde. Da einige naturalistische Menschenbilder die Autonomie des Menschen ausschlössen, liefen beide Konzepte sich gegenseitig ausschließend nebeneinander her. Kritisiert wurde zudem eine zu schwammige Lesart des Autonomie- und Selbstgesetzgebungsbegriffs von Kant. Der Begründungshergang war tatsächlich nicht ganz durchsichtig. Der Vortrag wies aber in eine Richtung, über die man weiter nachdenken sollte. Vielleicht gibt es die Möglichkeit, beide Beschreibungsebenen anzuerkennen und in einem Mehrebenen-Modell der menschlichen Natur zu integrieren.

\section{Transparenz in der Bioethik}

Roman Beck referierte im Anschluss über sein Dissertationsprojekt „Transparenz in der biomedizinischen Forschung". Beck unterschied zunächst zwischen Verfügungs- und Orientierungswissen (Mittelstraß 1982) und wies darauf hin, dass (natur-)wissenschaftliches Wissen nicht die einzige Wissensquelle der Biomedizin sei. Beck widmete sich dem Begriff der Transparenz und unterschied drei Arten: perzeptive, informationelle und kognitive Transparenz. Transparenz wurde von Beck also als ein komplexer Begriff definiert, der im deskriptiven Modus eine formelle und eine inhaltliche Komponente habe. Analog dazu orientierten sich Forderungen nach Transparenz im präskriptiven Modus an dieser Unterscheidung: Es seien dies dementsprechend die Forderungen nach relevanter, wahrhaftiger, vollständiger und verständlicher Information, sowie der Angemessenheit des sprachlichen Ausdrucks. Hier fänden sich informelle und formelle Aspekte wieder. Nun, so Beck, bestehe die Möglichkeit, die Transparenzpflicht aktiv oder passiv zu begründen, beides mit Rückgriff auf Kant. Die Transparenzforderung könne (in der aktiven Lesart) uminterpretiert werden als Hilfsforderung in Notsituationen. Das ließe eine
Lesart im Lichte des kategorischen Imperativs zu. Die Maxime, Menschen in Notsituationen zu helfen, könne durchaus zu einem allgemeinen Gesetz erhoben werden. Im Gegensatz zu anderen gesellschaftlichen Systemen, wo andere Güter und Pflichten gerade dazu beitragen könnten, die Transparenzpflicht fallen zu lassen (Politik - Stabilität, Wirtschaft - Kollegialität, o. ä.), stünden in der Wissenschaft stattdessen weitere Güter auf der Seite der Transparenzpflicht. Beck nannte zwar keine Beispiele, aber mit diesem Hinweis könnte eine Brücke zu den Prämissen vom Anfang des Vortrags geschlagen werden. Die Standortanalyse der Wissenschaft zu Beginn des Vortrags spiegelte nicht ganz die komplexen Strukturen wider, in denen die Wissenschaft in der Gesellschaft eingebettet ist. Kants Ethik als Fundament zur Begründung der Transparenzforderung heranzuziehen, entpuppte sich dennoch als lohnenswerter Ansatz. Der Aspekt der Zugänglichkeit von Information blieb als formeller Aspekt der Transparenzpflicht aber unberücksichtigt.

\section{Epigenetik}

Sebastian Schuol stellte die Ergebnisse aus seinem Dissertationsprojekt „Konsequenzen eines erweiterten Genbegriffs. Eine Untersuchung der theoretischen Grundlagen einer Ethik der Genetik aus der Perspektive der Epigenetik" vor. Die Epigenetik eröffne ein neues Verständnis von der ontologischen Grundeinheit der Vererbung: dem Gen. Schuol sagte zu dieser Entwicklung, dass seit dem Human Genome Project nicht mehr die Basensequenz als die Grundeinheit der Vererbung angesehen werden könne, sondern auch der regulativen „Zellmaschinerie“ große Bedeutung beigemessen werden müsse. Die Epigenetik zeige, dass regulative Strukturen der DNA in Interaktion mit ihrer Umwelt ausgebildet werden. Er sieht es als brisant an, dass diese für die Entwicklung entscheidenden Metainformationen auch transgenerational vererbt werden könnten.

So rekonstruierte Schuol zunächst historisch die Veränderung des Genbegriffs, die sich in drei Stufen vollzogen habe. Dieser Dreiklang stelle eine ontologische Wende in der Betrachtung des Gens dar. Schuol vertrat die These, dass die Bioethik dieser ontologischen Wende hinterherhinke 
und sich noch immer am klassischen Genbegriff abarbeite. Er verwies auf den Genetiker Conrad Hal Waddington (1905-1975), der die Phänotypentwicklung bereits ,auf dem Schirm“ hatte und daraus einen systemischen Genbegriff entwickelte. Dieser systemische Genbegriff hebe die strikte Trennung zwischen „Innen“ und „Außen“ der Gene auf, zugunsten eines eher monistischen Verständnisses von Genen und ihrer Umwelt.

Im zweiten Teil seines Vortrags präsentierte er eine kurze Analyse mehrerer Artikel des Spiegels. Die öffentliche Debatte propagiere vor allem eine „Eigenverantwortung der Mutter" auf Basis der neuesten Ergebnisse der Epigenetik. Die Abkehr vom Genfatalismus und die Erhöhung der Eigenverantwortung der Mutter bei der Schwangerschaft betrachtete Schuol daraufhin kritisch. Er meinte, eine gesteigerte Eigenverantwortung der Mütter könne aus den Ergebnissen der Epigenetik nicht abgeleitet werden. Es sei ebenso gut möglich, daraus eine gesamtgesellschaftliche Verantwortung zu begründen. Dem kann man sich anschließen. Eine Berücksichtigung der Debatte um den „naturalistischen Fehlschluss" wäre am Ende des Vortrags lohnenswert gewesen (Engels 1993).

\section{Authentizität als Maßstab und Herausforderung}

Der Titel von Jon Leefmanns Präsentation lautete: „Gibt es ein nicht-reduzierbares Authentizitätsargument für die Diskussion um das (Neuro)Enhancement?“. Leefmann versuchte zunächst Authentizität von Aufrichtigkeit abzugrenzen. Unter Authentizität verstand er eine Kongruenz zwischen innerer Selbstbeschreibung und äußerer Zuschreibung. Wohingegen die Aufrichtigkeit lediglich eine Fremdzuschreibung sei. Leefmann erörterte daraufhin das Problem, wie Neuro-Enhancement das Kongruenzverhältnis der Authentizität stören könne. Den Thesen von Carl Elliott (2003) hielt er entgegen, dass es kein essentielles Selbst gibt. Gäbe es ein essentielles Selbst, wäre es subjektiv nicht zugänglich, da die Essenz ein Begriff sei, der sich lediglich aus einer intersubjektiven Perspektive entwickeln lasse. Eine essentialistische Authentizitätskonzeption sei inkonsistent, aber eine Konzeption, die Authentizität als Kongruenzbegriff versteht, könne eine neue Perspek- tive auf die Neuro-Enhancement-Debatte werfen, so Leefmann. Ob man diese Debatte nicht einfach als Frage nach der Möglichkeit der ,rationalen Selbstbestimmung" verstehen könne, ob das nicht das Gleiche sei, müsse näher untersucht werden. Die Grenzziehung von illegitimem und legitimem Überstrapazieren des Verhältnisses von Selbstbeschreibung und Außenzuschreibung könnte ein Problem dieses Ansatzes sein. Der Vortrag war kompliziert, aber spannend! Diesen Ansatz sollte man unbedingt weiter verfolgen. Ein Vergleich mit dem Fazit in Oliver Müllers Buch „Zwischen Mensch und Maschine. Vom Glück und Unglück des Homo-Faber“" würde lohnen (Müller 2010).

\section{Darwin und die frühe Eugenik-Debatte}

In einem langen und sehr materialreichen Vortrag befasste sich Eve-Marie Engels mit dem Thema „Charles Darwins Auseinandersetzung mit zeitgenössischen Ideen der Selbstgestaltung". Engels setze sich darin mit Darwins Werk und seiner frühen Rezeption auseinander. Dabei ließ sie auch die Briefwechsel nicht unberücksichtigt. Besonderes Augenmerk richtete Engels auf die DarwinInterpretationen von William R. Greg und Francis Galton. Diese Zeitgenossen Darwins seien der Auffassung gewesen, die künstliche Selektion beeinflusse die natürliche Selektion in negativer Weise, sprich: Künstliche Selektion sei der Grund dafür, dass es so viele kranke, schwache und arme Menschen gebe. Nach der Auffassung dieser Eugeniker, so referierte Engels, war die passive Eugenik - also die Hilfsbereitschaft gegenüber diesen Bevölkerungsgruppen - verantwortlich für die Schwäche der Gesamtzivilisation. Sie plädierten daher für eine aktive Eugenik, die die menschliche Entwicklung wieder auf den Pfad der natürlichen Selektion bringen sollte. Greg und Galton hätten dafür die Geburtenkontrolle durch Verhütungsmittel empfohlen. Darwin sei dieser Maßnahme gegenüber aus zwei Gründen skeptisch gewesen. Zum einen sei er skeptisch gewesen, weil er nicht recht wusste, wie man einen Kriterienkatalog begründen könne, der festlegt, wer sich fortpflanzen dürfe und wer nicht. Zum anderen glaubte er, dass die erhöhte Zugänglichkeit dazu führe, dass sich die Sexualmoral zuungunsten der Familienbande entwickeln würde. Er 
hatte, so Engels, Angst, dass die Sexualmoral so verrohe, dass es nicht mehr zu Familiengründungen käme und bestehende Familien zerbrächen.

Engels stellte die Vermutung an, Darwin hätte, da er zeitlebens krank war und eine entfernte Verwandte geheiratet hatte, Angst gehabt, der Kriterienkatalog könne auf ihn zutreffen. Er habe aus diesen Gründen die aktive Eugenik durch Verhütungsmittel abgelehnt.

Insgesamt wurden auf der Abschlusstagung des Graduiertenkollegs einige hochinteressante Vorträge präsentiert, die auf die hohe Qualität der Projekte, die am Kolleg durchgeführt wurden, schließen lassen. An diese Beobachtung schließt sich die Hoffnung an, dass nach dem Auslaufen der Fördergelder der Deutschen Forschungsgemeinschaft ein angemessener Ersatz für das Graduiertenkolleg gefunden wird, um sich weiterhin interdisziplinär den wachsenden Herausforderungen der Biowissenschaft zu widmen.

\section{Literatur}

Elliott, C., 2003: Better than Well. American Medicine Meets the American Dream. New York

Engels, E.-M., 1993: George Edward Moores Argument der „naturalistic fallacy“ in seiner Relevanz für das Verhältnis von philosophischer Ethik und empirischen Wissenschaften. In: Eckensberger, L.H.; Gähde, U. (Hg.): Ethische Norm und empirische Hypothese. Frankfurt a. M., S. 92-132

Grunwald, A., 2008: Auf dem Weg in eine nanotechnologische Zukunft. Philosophisch-ethische Fragen. Freiburg i. Br.

Habermas, J., 2002: Die Zukunft der menschlichen Natur. Auf dem Weg zu einer liberalen Eugenik? Frankfurt a. M; http://www.gbv.de/dms/faz-rez/FD 1200409272491186.pdf (download 19.6.13)

Janich, P., 2009: Kein neues Menschenbild. Zur Sprache der Hirnforschung. Frankfurt a. M.

Lenk, H., 1991: Zu einer praxisnahen Ethik der Verantwortung in den Wissenschaften. In: Lenk, H. (Hg.): Wissenschaft und Ethik. Stuttgart, S. 54-75

Mittelstraß, J., 1982: Wissenschaft als Lebensform. Zur gesellschaftlichen Relevanz und zum bürgerlichen Begriff der Wissenschaft. In: Mittelstraß, J. (Hg.): Wissenschaft als Lebensform. Reden über philosophische Orientierungen in Wissenschaft und Universität. Frankfurt a. M., S. 11-36
Müller, O., 2010: Zwischen Mensch und Maschine Vom Glück und Unglück des Homo faber. Berlin

Patzig, G., 1988: Der kategorische Imperativ in der Ethik-Diskussion der Gegenwart. In: Patzig, G. (Hg.): Tatsachen, Normen, Sätze. Aufsätze und Vorträge. Stuttgart, S. 155-177

Saage, R., 2006: Konvergenztechnologische $\mathrm{Zu}-$ kunftsvisionen und der klassische Utopiediskurs. In: Nordmann, A. (Hg.): Nanotechnologien im Kontext. Philosophische, ethische und gesellschaftliche Perspektiven. Berlin, S. 179-194

$\ll »$

\section{Real-Time Technology Assessment in der Wüste}

\section{Winter School zu „Anticipatory Governance of Emerging Technologies“}

Mesa, Arizona, USA, 3.-10. Januar 2013

\section{von Stefanie B. Seitz, ITAS}

Nur eine Stunde Fahrt nordöstlich vom Zentrum der Stadt Phönix befindet man sich mitten in der Sonora Wüste und damit in einer völlig anderen Welt: verschwunden die spiegelnden Hochausfassaden und perfekt gepflegten Grünflächen des Arizona State University (ASU) Campus in Tempe, dafür haushohe Kandelaber-Kakteen, das Wahrzeichen dieser Landschaft und namensgebend für die 1930 erbaute Saguaro Lake Ranch, die in Arizona ,wirklich historisch" ist, wie man uns stolz versicherte. Die Ranch liegt umgeben von der malerischen Landschaft des Tonto National Forest am Salt River, nur einen Steinwurf weit entfernt vom Stewart Mountain-Damm, der diesen zum Saguaro Lake aufstaut. Ursprünglich diente die Ranch als Speisehalle für die Bauarbeiter jenes Dammes, doch vom 3.-10. Januar 2013 beherbergte das heutige Feriendomizil vierzehn Nachwuchswissenschaftler verschiedener Disziplinen, die aus aller Welt angereist waren, um an der, von Dave Guston organisierten, Winter School zum Thema „Anticipatory Governance of Emerging Technologies" teilzunehmen und dabei akademische Auslandserfahrungen der besonderen Art sammeln zu können. 